\title{
Circulating IL-6 concentrations link tumour necrosis and systemic and local inflammatory responses in patients undergoing resection for colorectal cancer
}

\author{
G J K Guthrie*,1, C S D Roxburgh ${ }^{1}$, C H Richards ${ }^{1}$, P G Horgan ${ }^{1}$ and D C McMillan ${ }^{1}$ \\ ${ }^{1}$ Academic Unit of Surgery, School of Medicine, University of Glasgow, Royal Infirmary, Glasgow, G31 2ER, UK
}

Background: Cancer-associated inflammation, in the form of local and systemic inflammatory responses, appear to be linked to tumour necrosis and have prognostic value in patients with colorectal cancer. However, their relationship with circulating biochemical mediators is unclear. The aim of the present study was to examine the interrelationships between circulating mediators, in particular interleukin-6 (IL-6) and tumour necrosis, and local and systemic inflammatory responses in patients undergoing resection for colorectal cancer.

Methods: Data were collected from preoperative blood tests for 118 patients who underwent resection for colorectal cancer. Analysis of circulating IL-6, IL-10, vascular endothelial growth factor (VEGF), differential white cell count, C-reactive protein, and albumin were carried out. Routine pathology specimens were examined for tumour characteristics including necrosis and the extent of the inflammatory cell infiltrate. Body composition was examined using body mass index (BMI), total body fat, subcutaneous body fat, visceral fat, and skeletal muscle mass.

Results: Circulating IL-6 concentrations were significantly associated with increased T stage $(P<0.05)$, tumour necrosis $(P<0.001)$, IL-10 ( $P<0.001)$, VEGF $(P<0.001)$, modified Glasgow Prognostic Score (mGPS; $P<0.001)$, white cell $(P<0.01)$ and platelet $(P<0.01)$ counts, and low skeletal muscle index $(P<0.01)$. When normalised for T stage, tumour necrosis was associated with IL-6 $(P<0.001)$, IL-10 $(P<0.01)$, VEGF $(P<0.001)$, mGPS $(P<0.001)$, neutrophil-lymphocyte ratio (NLR; $P<0.05)$, white cell $(P<0.001)$, neutrophil $(P<0.05)$, and platelet counts $(P<0.005)$, and skeletal muscle index $(P<0.001)$.

Conclusion: The present study provides, for the first time, supportive evidence for the hypothesis that tumour necrosis, independent of T stage, is associated with elevated circulating IL-6 concentrations, thereby modulating both local and systemic inflammatory responses including angiogenesis that, in turn, may promote tumour progression and metastases.

Colorectal cancer is the second most common cause of cancer death in both men and women in the United Kingdom, with 16000 deaths per year (Cancerstats, 2005-2010). Despite improvements in treatment, outcomes remain poor, with approximately half of those undergoing curative resection dying from the disease (Oliphant et al, 2013).

In recent years, it has become increasingly apparent that cancerassociated inflammation, in the form of local and systemic inflammatory responses, is a key determinant of disease progression and survival in colorectal cancer. Indeed, there is consistent evidence that the presence of a high-grade inflammatory or immune cell infiltrate in the tumour and in the immediate microenvironment predicts survival independent of tumour stage in colorectal cancer (Klintrup et al, 2005; Galon et al, 2006; Roxburgh and Mcmillan, 2012). Furthermore, many studies have reported that markers of the systemic inflammatory response, in 
particular C-reactive protein, albumin, and their combination in the modified Glasgow Prognostic Score (mGPS), and the neutrophil-lymphocyte ratio (NLR) predict survival independent of tumour stage (Guthrie GJ et al, 2013a; Mcmillan, 2013).

Despite the strong evidence linking the local and systemic inflammatory responses to colorectal cancer survival and their apparent independent prognostic value, the mechanisms by which these two related inflammatory processes are activated, maintained, and interact are not clear. Recent work has highlighted tumour necrosis as a prognostic factor in colorectal cancer and its close associations with the local and systemic inflammatory responses (Pollheimer et al, 2010; Richards et al, 2012b). However, what remains unclear is the mechanism linking tumour necrosis and the local and systemic inflammatory responses.

One hypothesis is that tumour necrosis may link these two key inflammatory processes via immunological mediators, in particular interleukin-6 (IL-6), present in the tumour microenvironment and in the circulation (Trikha et al, 2003; Guthrie GJ et al, 2013b).

The molecular links between tumour necrosis and these key inflammatory processes are likely to be complex. The 'dirty', hypoxia-driven, unscheduled cell death occurring in tumour necrosis makes it likely that this process may stimulate a variety of inflammatory mediators that can influence both the systemic and local inflammatory responses. Other potential candidates for such mediators include circulating IL-10 and vascular endothelial growth factor (VEGF; Mocellin et al, 2005; Hsu and Chung, 2006).

Therefore, the aim of the present study was to examine whether circulating mediators, in particular IL-6, may be a link between tumour necrosis and local and systemic inflammatory responses in patients undergoing curative resection for colorectal cancer.

\section{PATIENTS AND METHODS}

Patients with colorectal cancer who, on the basis of preoperative staging and laparotomy findings, were considered to have undergone an elective, potentially curative resection of colorectal cancer between April 2004 and July 2009 in a single surgical unit at Glasgow Royal Infirmary were included in the study. Tumours were staged using the conventional tumour, node, metastasis (TNM) staging system, 7th edition, 2010 (AJCC, 2010). Patients with conditions known to elicit an acute or chronic systemic inflammatory response were excluded. These were namely (1) preoperative chemoradiotherapy, (2) clinical evidence of active preoperative infection, or (3) chronic active inflammatory diseases such as rheumatoid arthritis.

Blood samples were collected before surgery for routine laboratory analysis of full blood count, white cell and lymphocyte counts, albumin, and C-reactive protein.

The mGPS was constructed based on routine preoperative blood tests (Mcmillan, 2013). Briefly, patients with both an elevated C-reactive protein $\left(>10 \mathrm{mgl}^{-1}\right)$ and low albumin $\left(<35 \mathrm{gl}^{-1}\right)$ were allocated a score of 2 ; patients in whom only C-reactive protein was elevated $\left(>10 \mathrm{mgl}^{-1}\right)$ were allocated a score of 1 ; and patients with a normal C-reactive protein were allocated a score of 0 .

The NLR ratio was constructed using previously documented thresholds (Guthrie GJ et al, 2013a). Briefly, NLR was determined by dividing the absolute neutrophil count by the absolute lymphocyte count, and the NLR data were then dichotomised and given a score of $0(<5: 1)$ and $1(>5: 1)$.

The study was approved by the Research Ethics Committee, Glasgow Royal Infirmary, Glasgow.

Blood samples were centrifuged and the serum stored at $-80{ }^{\circ} \mathrm{C}$ before analysis of IL-6, IL-10, and VEGF. Circulating concentrations of IL-6, IL-10, and VEGF were measured using commercially available human colorimetric enzyme-linked immunosorbent assays (Quantikine ELISA, R\&D Systems, Europe Ltd, Abingdon, UK). The minimum detectable concentrations were $2 \mathrm{pg} \mathrm{ml}^{-1}$ for IL-6, $4 \mathrm{pg} \mathrm{ml}^{-1}$ for IL-10, and $9 \mathrm{pg} \mathrm{ml}^{-1}$ for VEGF.

Assessment of the local inflammatory cell infiltrate and tumour necrosis was performed on original haematoxylin and eosinstained full sections of the tumour, felt to represent the maximum depth of tumour invasion. The local inflammatory response was evaluated previously in this cohort using the method described by Klintrup and Makinen (K-M; Klintrup et al, 2005). Briefly, the $\mathrm{K}-\mathrm{M}$ criterion is a four-point scale with scores as follows: a score of 0 indicates no increase in inflammatory cells at the invasive margin, a score of 1 indicates presence of a mild/patchy increase in inflammatory cell reaction at the invasive margin but no destruction of invading cancer cell islets, a score of 2 indicates observation of a band-like inflammatory reaction at the invasive margin, and a score of 3 indicates observation of a florid inflammatory reaction with cup-like inflammatory infiltrate at the invasive margin. The scores were then dichotomised to 'high' and 'low' grade inflammation in line with the previously published literature (Klintrup et al, 2005).

Tumour necrosis was assessed using the method described by Pollheimer et al (2010). Briefly, at $\times 40$ magnification, the full sections were examined for evidence of tumour necrosis. Tumour necrosis was graded as 'absent' (none), 'focal' $(<10 \%$ of tumour surface area), 'moderate' (10-30\% tumour surface area), or 'extensive' ( $>30 \%$ of tumour surface area) in each section before an assessment of overall extent of necrosis was made.

To test the reliability of the evaluation of necrosis, sections of 30 patients (average of 3 slides per patient) were examined independently by two observers (GJKG and CSDR) blinded to clinical outcome and clinicopathological variables. The intraclass correlation coefficient (ICC) for the assessment of local inflammatory cell infiltrate was 0.81 and for tumour necrosis was 0.70 .

To evaluate metabolic upset, the body composition parameters, body mass index (BMI), total body fat, subcutaneous body fat, visceral fat, and skeletal muscle mass, as previously described (Richards et al, 2012c), were used to assess the relationship between systemic and local inflammation, tumour necrosis and circulating immunological factors, and cancer cachexia.

Statistics. Data are presented as median and range. Grouping of variables was carried out using standard thresholds for laboratory parameters. As there are no widely accepted thresholds for IL-6, IL-10, and VEGF, the values were grouped as tertiles (Lee et al, 2012). The concentrations of IL-6, IL-10, and VEGF below the threshold of sensitivity of the respective assays were expressed as equal to this threshold. The relationships between the groups of patients was carried out using Mantel-Haenszel $\left(\chi^{2}\right)$ test for trend and the Kruskal-Wallis test and Spearman's rank correlation as appropriate. The relationship between IL-6 and cancer-specific survival was examined using the Kaplan-Meier method. Analysis was performed using SPSS software version 19 (SPSS Inc., Chicago, IL, USA).

\section{RESULTS}

The majority of patients were $\geqslant 65$ years old $(66 \%)$, were male (51\%), had TNM stage I/II disease (62\%), and the majority had C-reactive protein $(68 \%)$ and albumin $(72 \%)$ concentrations in the normal range and a normal mGPS (68\%). Of the 33 patients with hypoalbuminaemia, 16 (48\%) had an elevated C-reactive protein. The majority of patients also had total white cell counts (71\%), neutrophil counts (89\%), lymphocyte counts (82\%), and platelet counts $(87 \%)$ in the normal range and a normal NLR (80\%). 
Serum IL-6 concentrations were divided into tertiles. The tertiles had approximately equal numbers of patients: tertile 1 (39 patients), tertile 2 (39 patients), and tertile 3 (40 patients). Tertile 1 (median concentration $2 \mathrm{pg} \mathrm{ml}^{-1}$, range $0.8-3.38$ ) the lowest, tertile 2 moderately elevated concentration (median concentration $4.85 \mathrm{pg} \mathrm{ml}^{-1}$, range $3.43-6.01$ ), and tertile 3 (median concentration (9.97 $\mathrm{pg} \mathrm{ml}^{-1}$, range 6.1-252.46) the highest.

On assessment of tumour necrosis, $48 \%$ had no evidence of tumour necrosis, $29 \%$ had focal areas of tumour necrosis, $11 \%$ had moderate tumour necrosis, whereas $12 \%$ had extensive tumour necrosis. On assessment of the local tumour inflammatory cell response using the $\mathrm{K}-\mathrm{M}$ criteria, the majority of patients were considered to have a high-grade inflammatory cell infiltrate (55\%).

On Spearman's rank correlation of individual values, there were significant associations between circulating concentrations of IL-6 and IL-10 $(r=0.56, P<0.001)$, C-reactive protein $(r=0.45$, $P<0.005)$, albumin $(r=-0.65, P<0.001)$, and the skeletal muscle index $(r=-0.38, P=0.056)$. Circulating concentrations of IL-10 were significantly associated with albumin $(r=0.40, P<0.05)$. Circulating concentrations of VEGF were significantly associated with C-reactive protein $(r=0.32, P<0.05)$.

The relationship between circulating IL-6 tertiles, tumour characteristics, and systemic responses in all patients with colorectal cancer is shown in Table 1 . When all patients were considered, circulating IL-6 was associated with gender $(P<0.05)$, increased IL-10 $(P<0.001)$, VEGF $(P<0.001)$, tumour site

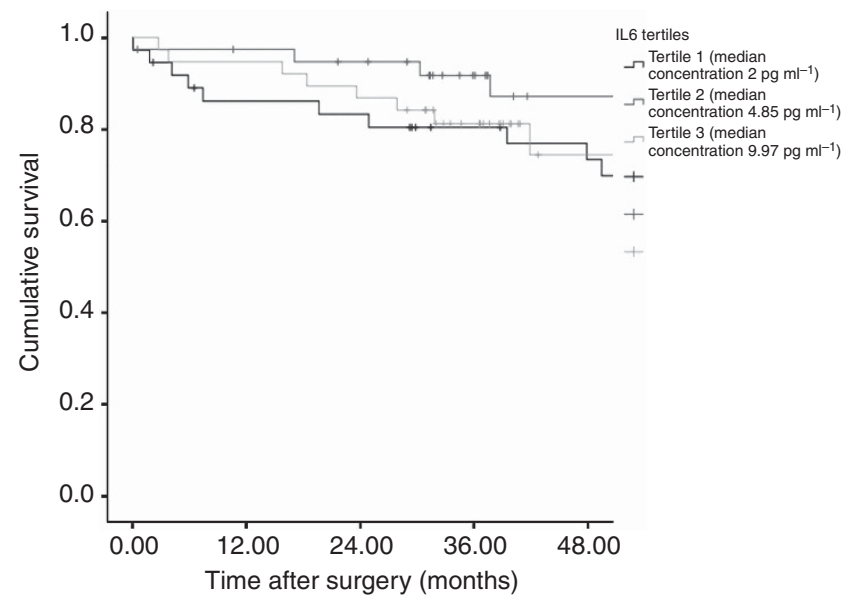

Figure 1. Kaplan-Meier analysis of cancer survival in relation to serum IL-6 concentration ( $P=0.292)$.

Table 1. The relationships between circulating IL-6, tumour characteristics, and systemic responses in patients with colorectal cancer

Circulating IL-6 $\left(\mathrm{pg} \mathrm{ml}^{-1}\right)$

\begin{tabular}{|c|c|c|c|c|}
\hline Patient characteristics & $\begin{array}{c}<3.4 \mathrm{pg} \mathrm{ml}^{-1} \\
(n=39)\end{array}$ & $\begin{array}{c}3.4-6.0 \mathrm{pg} \mathrm{ml}^{-1} \\
(n=39)\end{array}$ & $\begin{array}{c}>6.0 \mathrm{pg} \mathrm{ml}^{-1} \\
(n=40)\end{array}$ & $\boldsymbol{P}$-value \\
\hline Age $(<65 / 65-74 / \geqslant 75$ years $)$ & $18 / 12 / 10$ & $13 / 16 / 18$ & $8 / 11 / 12$ & 0.080 \\
\hline Sex (male/female) & $25 / 14$ & $20 / 19$ & $15 / 25$ & 0.019 \\
\hline
\end{tabular}

Tumour characteristics

Presentation (elective/emergency)

Tumour site (colon/ rectum)

T stage $\left(T_{1} / T_{2} / T_{3} / T_{4}\right)$

$N$ stage $\left(\mathrm{N}_{0} / \mathrm{N}_{1} / \mathrm{N}_{2}\right)$

Tumour necrosis (low/high)

Vascular invasion (yes/no)

Inflammatory cell infiltrate (K-M low grade/high grade)

$36 / 1$

$17 / 21$

$4 / 7 / 23$

$29 / 4 / 6$

$24 / 13$

$14 / 25$

$19 / 20$
$37 / 0$

$21 / 17$

$2 / 5 / 25 / 7$

$23 / 12 / 4$

$24 / 12$

$19 / 20$

$18 / 19$

\begin{tabular}{l|l}
$32 / 1$ & 0.948
\end{tabular}

\begin{tabular}{l|l}
$24 / 10$ & 0.028
\end{tabular}

$1 / 2 / 25 / 12$

$21 / 14 / 5$

$6 / 34$

$14 / 26$

$13 / 24$
0.028

0.007

0.237

$<0.001$

0.927

0.239

\section{Circulating mediators}

IL-10 (tertiles)

VEGF (tertiles $\mathrm{pg} \mathrm{ml}^{-1}$ )

$19 / 12 / 7$

$25 / 0 / 0$

$11 / 12 / 7$

$10 / 29 / 0$

$5 / 9 / 20$

$0 / 5 / 35$

$<0.001$

Systemic responses

Systemic inflammatory response (mGPS 0/1/2)

NLR (low $<5 /$ high $>5$ )

Anaemia

$>13 \mathrm{~g} \mathrm{dl}^{-1}$ (men), $>11.5 \mathrm{~g} \mathrm{dl}^{-1}$ (women)

$<13 \mathrm{~g} \mathrm{dl}^{-1}$ men, $<11.5 \mathrm{~g} \mathrm{dl}^{-1}$ (women)

White cell count $\left(<8.5 / 8.5-11.5 />11.5 \times 10^{9}\right.$ per I)

Neutrophil count $\left(<7.5 />7.5 \times 10^{9}\right.$ per l)

Lymphocyte count $\left(\geqslant 1 /<1 \times 10^{9}\right.$ per I)

Monocyte count $\left(<1 />1 \times 10^{9}\right.$ per I)

Platelets $\left(\leqslant 400 />400 \times 10^{6}\right.$ per l)

BMI ( $\mathrm{kg} \mathrm{m}^{-2}$; normal weight, overweight, obese)

Total fat index $\left(\mathrm{cm}^{2} \mathrm{~m}^{-2}\right.$; low/medium/high)

Subcutaneous fat index $\left(\mathrm{cm}^{2} \mathrm{~m}^{-2} ;\right.$ low/medium/high)

Visceral fat index $\left(\mathrm{cm}^{2} \mathrm{~m}^{-2}\right.$; low/medium/high)

Skeletal muscle index $\left(\mathrm{cm}^{2} \mathrm{~m}^{-2}\right.$; low/medium/high)

\begin{tabular}{c|c}
\hline $34 / 4 / 1$ & $31 / 8 / 0$ \\
$34 / 5$ & $30 / 9$ \\
$12 / 27$ & $13 / 26$ \\
& \\
& \\
$30 / 6 / 3$ & $32 / 6 / 1$ \\
$35 / 4$ & $38 / 1$ \\
$6 / 33 / 0$ & $9 / 28 / 2$ \\
$38 / 1$ & $34 / 5$ \\
$37 / 2$ & $35 / 4$ \\
$1 / 6 / 2$ & $6 / 2 / 5$ \\
$0 / 3 / 6$ & $6 / 4 / 3$ \\
$0 / 4 / 5$ & $6 / 3 / 4$ \\
$1 / 5 / 3$ & $6 / 6 / 1$ \\
$0 / 1 / 8$ & $2 / 5 / 6$
\end{tabular}

$15 / 10 / 15$

$30 / 10$

$11 / 29$

$22 / 11 / 7$

$32 / 8$

$4 / 36 / 0$

$34 / 6$

$31 / 9$

$8 / 7 / 8$

$8 / 6 / 9$

$11 / 2 / 10$

$8 / 7 / 8$

$7 / 10 / 6$ 
Table 2. The interrelationships between different pathological and clinical parameters in patients with T3 stage disease undergoing potentially curative resection for colorectal cancer $(n=73)$

\begin{tabular}{|c|c|c|c|c|c|c|c|c|c|}
\hline & \multicolumn{2}{|c|}{ Tumour characteristics } & \multicolumn{3}{|c|}{ Circulating mediators } & \multicolumn{4}{|c|}{ Systemic responses } \\
\hline & $\begin{array}{l}\text { Tumour } \\
\text { necrosis }\end{array}$ & $\begin{array}{c}\text { K-M } \\
\text { (low/high grade) }\end{array}$ & IL-6 & IL-10 & VEGF & $\begin{array}{c}\text { Platelet } \\
\text { count }\end{array}$ & mGPS & NLR & $\begin{array}{c}\text { Skeletal } \\
\text { muscle } \\
\text { index }\end{array}$ \\
\hline Site & 0.111 & 0.787 & 0.023 & 0.644 & 0.008 & 0.173 & 0.042 & 0.868 & 0.427 \\
\hline Tumour necrosis & & 0.140 & $<0.001$ & 0.008 & $<0.001$ & 0.004 & $<0.001$ & 0.044 & 0.009 \\
\hline K-M (low/high grade) & & & 0.768 & 0.837 & 0.428 & 0.719 & 0.320 & 0.886 & 0.496 \\
\hline IL-6 & & & & $<0.001$ & $<0.001$ & 0.038 & $<0.001$ & 0.301 & 0.133 \\
\hline IL-10 & & & & & 0.004 & 0.222 & 0.017 & 0.904 & 0.297 \\
\hline VEGF & & & & & & 0.017 & $<0.001$ & 0.161 & 0.195 \\
\hline Platelet count & & & & & & & 0.001 & 0.619 & 0.537 \\
\hline mGPS & & & & & & & & 0.339 & 0.600 \\
\hline
\end{tabular}

$(P<0.05)$, increased $\mathrm{T}$ stage $(P<0.01)$, tumour necrosis $(P<0.001)$, increased mGPS $(P<0.001)$, increased white cell $(P<0.05)$ and platelet $(P<0.05)$ counts, and low skeletal muscle index $(P<0.01)$.

When all patients were considered together, tumour necrosis was associated with increased $\mathrm{T}$ stage $(P<0.005)$, decreased local inflammatory cell infiltrate $(P<0.05)$, increased IL-6 $(P<0.001)$, increased IL-10 $(P<0.005)$, increased VEGF $(P<0.001)$, mGPS $(P<0.001)$, anaemia $(P<0.05)$, elevated white cell $(P<0.005)$, neutrophil count $(P<0.05)$, and platelet counts $(P<0.001)$, and skeletal muscle index $(P<0.001)$.

In the present study, the median follow-up for the survivors was 53 (range 25-91) months. During this period, 29 patients died from colorectal cancer and 11 from other causes. Neither IL- 6 and IL-10 nor VEGF were significantly associated with cancer-specific survival (Figure 1).

In order to account for the effect of $\mathrm{T}$ stage, the relationship between tumour characteristics, circulating mediators, and systemic responses in patients with stage T3 colorectal cancer $(n=73)$ was examined (Table 2 ). When T3 colon tumours were considered alone, the relationships between IL-6 and IL-10 $(P<0.001)$, VEGF $(<0.001)$, mGPS $(P<0.001)$, platelet count $(P<0.05)$, and tumour necrosis $(P<0.001)$ remained significant.

\section{DISCUSSION}

The results of the present study show that tumour necrosis, independent of tumour size, was significantly associated with elevated concentrations of IL-6, IL-10, VEGF, mGPS, NLR, platelet count, and the skeletal muscle index. Furthermore, IL-6 concentrations were significantly associated with IL-10, VEGF, platelet counts, mGPS, and the skeletal muscle index. In addition, IL-10 and VEGF were significantly associated with each other and both were significantly associated with systemic inflammation, as evidenced by the mGPS. Taken together, the results of the present study indicate that IL-6 is indeed a plausible mediator of the relationship between tumour necrosis and systemic inflammatory responses in patients with operable colorectal cancer.

In the present study, circulating IL-6 concentrations were not significantly associated with the extent of the general local inflammatory cell infiltrate as evidenced by K-M criteria or cancer-specific survival. This does not preclude IL-6 concentrations being associated with specific inflammatory cell types. Also, the relatively small number of cancer deaths limits the conclusions that can be made about the prognostic value of IL-6. Furthermore, although most inflammatory cell types increase with a high K-M grade, macrophage counts have recently been reported to be similar in both low- and high-grade K-M grades (Richards et al, 2012a). It was therefore of interest that circulating concentrations of IL-6 were directly associated with IL-10 as tumour IL-10 levels can be sustained by the production of IL- 6 by tumour-infiltrating macrophages (Herbeuval et al, 2004; Mocellin et al, 2005). Although the exact mechanism by which IL-10 exerts its effect in the tumour microenvironment is unclear, it is accepted that this cytokine has multiple stimulatory and inhibitory effects on innate and adaptive immune responses respectively. Indeed, it has been proposed that IL-10 is a key anti-inflammatory cytokine involved in the switch from Th1 to Th2 immune responses (De Vita et al, 2000).

Therefore, it may be the balance between IL- 6 and IL-10 in the tumour microenvironment that may be important in determining the nature of local and systemic inflammatory responses in colorectal cancer.

Recent work has highlighted a direct relationship between the systemic inflammatory responses (mGPS) and the loss of skeletal muscle in patients with colorectal cancer (Richards et al, 2012c). In the present study, although there was a relatively small number of observations, there was also an association between elevated concentrations of circulating IL-6 and a low skeletal muscle mass. These results are consistent with work in experimental models of cancer cachexia that have implicated proinflammatory cytokines and their downstream signalling cascades in cancer-associated muscle wasting (Strassmann et al, 1992; Argiles et al, 2005; Bonetto et al, 2012). Prominent among these is the IL-6/JAK/STAT3 cascade (Bonetto et al, 2012) and therefore confirms the plausibility of the present hypothesis in patients with colorectal cancer.

The mechanism whereby tumour necrosis results in an increase in circulating IL-6 concentrations is not clear. One plausible hypothesis is that hypoxic tumour cells activate hypoxia-inducible factors that result in the production of IL-6 and VEGF and that these spill into the circulation raising circulating concentrations (see Figure 2). However, it is of interest that IL-6 concentrations do not appear to normalise following potentially curative resection of 


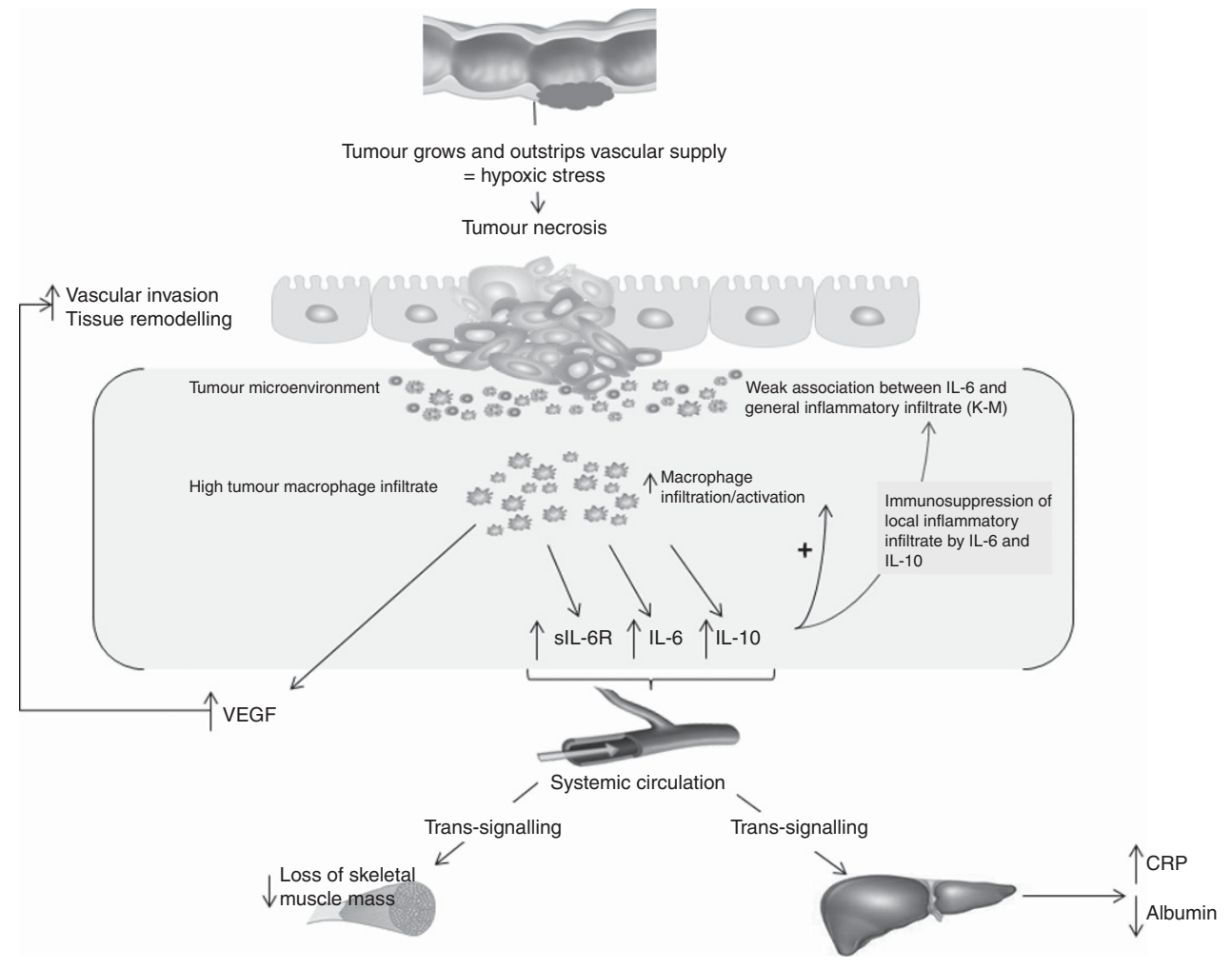

Figure 2. Proposed pathway linking tumour necrosis, IL-6, and systemic and local inflammatory responses in patients with colorectal cancer.

tumours, similar to C-reactive protein. Thus, it is also plausible that IL- 6 concentrations may be determined by tissues other than that of the tumour (Guthrie GJ et al, 2013b). Indeed, it has been reported that tumour-associated macrophages (TAMs) may play an important role, as they are known to produce significant amounts of IL- 6 and are reported to be a major source of IL-6 in both the serum and tumour microenvironment of patients with colorectal cancer (Lieubeau et al, 1999; Piancatelli et al, 1999). Indeed, numerous studies have reported the presence of macrophages in the tumour microenvironment (Roxburgh and Mcmillan, 2012). Despite this, the prognostic value of tumourinfiltrating macrophages remains unclear. At least nine studies have previously reported a relationship between TAMs and survival. Of these, five studies reported a strong association between the density of macrophages at the invasive margin and survival, whereas the remainder reported no significant association with survival (Roxburgh and Mcmillan, 2012).

Although some studies have reported that high macrophage infiltration is associated with improved survival (Lackner et al, 2004; Forssell et al, 2007; Zhou et al, 2010; Algars et al, 2012; Edin et al, 2012; Kinouchi et al, 2012), recent work that has identified the two main subpopulations of macrophages present in the tumour microenvironment suggests a more complex relationship between macrophage infiltration and survival (Sica et al, 2006; Solinas et al, 2009). Indeed, it has been reported that the distribution of the M1 and M2 subpopulations of macrophages in the tumour microenvironment may influence survival and that these subpopulations may localise to different areas of the tumour depending on the prevailing conditions, including tissue hypoxia (Movahedi et al, 2010; Laoui et al, 2011; Richards et al, 2012d). Indeed, it has long been recognised that TAMs localise to hypoxic regions of the tumour microenvironment (Harris, 2002). Therefore, it is plausible that the relative density of macrophages (perhaps M2) are important in such cytokine alterations and the elaboration of the systemic inflammatory response in patients with colorectal cancer and this requires further investigation.
Interestingly, recent work by Kantola et al (2012) and Guthrie and Mcmillan 2013) has highlighted the potential relationship between the systemic inflammatory response, as evidenced by mGPS, and alterations in a variety of serum cytokine concentrations and this, along with the results of the present study, may provide new insight into the inflammatory cells associated with the upregulation of the systemic inflammatory response in patients with colorectal cancer.

Indeed, with the exception of macrophages, few inflammatory cells can produce such a spectrum of cytokines and growth factors, and this is consistent with recent reports that macrophages are abundant in tumour microenvironments even in the absence of other inflammatory cells (Mohammed et al, 2012; Richards et al, 2012a). Therefore, the above findings are also consistent with the hypothesis that tumour necrosis (increasing with T stage) and IL-6 play an important role in linking local and systemic inflammatory responses in patients with colorectal cancer (Richards et al, 2012b).

It has also previously been suggested that IL-6 may stimulate systemic inflammatory responses through the trans-signalling pathway involving the soluble IL-6 receptor (Scheller et al, 2006; Rose-John et al, 2007), and it is plausible that intratumoural and circulating macrophages may contribute to this trans-signalling pathway (Scheller et al, 2006; Rose-John et al, 2007), and therefore play a key role in the evolution of a chronic systemic inflammatory response. Indeed, Chua et al (2011) proposed a similar scheme that proinflammatory cytokines may modulate both the local tumour microenvironment and a chronic systemic inflammatory response that affects normal organs, including liver and muscle. Irrespective, the relationship between this 'IL-6 trans-signalling' pathway and both local and systemic inflammatory responses in patients with colorectal cancer also merits further evaluation.

In summary, the present study therefore provides, for the first time, supportive evidence for the hypothesis that tumour necrosis, independent of T stage, elevates circulating IL-6 concentrations, thereby modulating both local and systemic inflammatory responses including angiogenesis that, in turn, may promote 
tumour progression and metastases. Further evaluation of the relationships between cells that produce IL-6 (e.g., macrophages) in the tumour microenvironment and in the circulation is of considerable interest.

\section{ACKNOWLEDGEMENTS}

We acknowledge the support and assistance of Professor A Michael Wallace and Dr Fiona Breckenridge.

\section{REFERENCES}

AJCC (2010) Colon and Rectum Cancer Staging. AJCC Cancer Staging Manual. AJCC Cancer Staging Manual. 7th edition.

Algars A, Irjala H, Vaittinen S, Huhtinen H, Sundstrom J, Salmi M, Ristamaki R, Jalkanen S (2012) Type and location of tumor-infiltrating macrophages and lymphatic vessels predict survival of colorectal cancer patients. Int $J$ Cancer 131: 864-873.

Argiles JM, Busquets S, Lopez-Soriano FJ (2005) The pivotal role of cytokines in muscle wasting during cancer. Int J Biochem Cell Biol 37: 2036-2046.

Bonetto A, Aydogdu T, Jin X, Zhang Z, Zhan R, Puzis L, Koniaris LG, Zimmers TA (2012) JAK/STAT3 pathway inhibition blocks skeletal muscle wasting downstream of IL-6 and in experimental cancer cachexia. Am J Physiol Endocrinol Metab 303: E410-E421.

CancerStats (2005-2010) http://www.cancerresearchuk.org

Chua W, Charles KA, Baracos VE, Clarke SJ (2011) Neutrophil/lymphocyte ratio predicts chemotherapy outcomes in patients with advanced colorectal cancer. Br J Cancer 104: 1288-1295.

De Vita F, O. M, Galizia G, Romano C, Lieto E, Iodice P, Tuccillo C, Catalano G (2000) Serum interleukin-10 is an independent prognostic factor in advanced solid tumors. Oncol Rep 7: 357-418.

Edin S, Wikberg ML, Dahlin AM, Rutegard J, Oberg A, Oldenborg PA, Palmqvist R (2012) The distribution of macrophages with a M1 or M2 phenotype in relation to prognosis and the molecular characteristics of colorectal cancer. PLoS One 7: e47045.

Forssell J, Oberg A, Henriksson ML, Stenling R, Jung A, Palmqvist R (2007) High macrophage infiltration along the tumor front correlates with improved survival in colon cancer. Clin Cancer Res 13: 1472-1479.

Galon J, Costes A, Sanchez-Cabo F, Kirilovsky A, Mlecnik B, Lagorce-Pages C, Tosolini M, Camus M, Berger A, Wind P, Zinzindohoue F, Bruneval P, Cugnenc PH, Trajanoski Z, Fridman WH, Pages F (2006) Type, density, and location of immune cells within human colorectal tumors predict clinical outcome. Science 313: 1960-1964.

Guthrie GJ, Charles KA, Roxburgh CS, Horgan PG, Mcmillan DC, Clarke SJ (2013a) The systemic inflammation-based neutrophil-lymphocyte ratio: experience in patients with cancer. Crit Rev Oncol Hematol; e-pub ahead of print 16 April 2013; doi: 10.1016/j.critrevonc.2013.03.010.

Guthrie GJ, Mcmillan DC (2013) Comment on 'Stage-dependent alterations of the serum cytokine pattern in colorectal carcinoma'. Br J Cancer 108 : 1915-1916.

Guthrie GJ, Roxburgh CS, Horgan PG, Mcmillan DC (2013b) Does interleukin-6 link explain the link between tumour necrosis, local and systemic inflammatory responses and outcome in patients with colorectal cancer? Cancer Treat Rev 39: 89-96.

Harris AL (2002) Hypoxia-a key regulatory factor in tumour growth. Nat Rev Cancer 2: 38-47.

Herbeuval J-P, Lelievre E, Lambert C, Dy M, Genin C (2004) Recruitment of STAT3 for production of IL-10 by colon carcinoma cells induced by macrophage-derived IL-6. J Immunol 172: 4630-4636.

Hsu CP, Chung YC (2006) Influence of interleukin-6 on the invasiveness of human colorectal carcinoma. Anticancer Res 26: 4607-4614.

Kantola T, Klintrup K, Vayrynen JP, Vornanen J, Bloigu R, Karhu T, Herzig KH, Napankangas J, Makela J, Karttunen TJ, Tuomisto A, Makinen MJ (2012) Stage-dependent alterations of the serum cytokine pattern in colorectal carcinoma. Br J Cancer 107: 1729-1736.

Kinouchi M, Miura K, Mizoi T, Ishida K, Fujibuchi W, Sasaki H, Ohnuma S, Saito K, Katayose Y, Naitoh T, Motoi F, Shiiba KI, Egawa S, Shibata C, Unno M (2012) Infiltration of CD40-positive tumor-associated macrophages indicates a favorable prognosis in colorectal cancer patients.
Hepatogastroenterology 60(121); e-pub ahead of print 8 June 2013; doi: 10.5754/hge12372.

Klintrup K, Makinen JM, Kauppila S, Vare PO, Melkko J, Tuominen H, Tuppurainen K, Makela J, Karttunen TJ, Makinen MJ (2005) Inflammation and prognosis in colorectal cancer. Eur J Cancer 41: 2645-2654.

Lackner C, Jukic Z, Tsybrovskyy O, Jatzko G, Wette V, Hoefler G, Klimpfinger M, Denk H, Zatloukal K (2004) Prognostic relevance of tumour-associated macrophages and von Willebrand factor-positive microvessels in colorectal cancer. Virchows Arch 445: 160-167.

Laoui D, Van Overmeire E, Movahedi K, Van Den Bossche J, Schouppe E, Mommer C, Nikolaou A, Morias Y, de Baetselier P, Van Ginderachter JA (2011) Mononuclear phagocyte heterogeneity in cancer: different subsets and activation states reaching out at the tumor site. Immunobiology 216 1192-1202.

Lee JK, Bettencourt R, Brenner D, LE TA, Barrett-Connor E, Loomba R (2012) Association between serum interleukin-6 concentrations and mortality in older adults: the Rancho Bernardo study. PLoS One 7: e34218.

Lieubeau B, Heymann MF, Henry F, Barbieux I, Meflah K, Gregoire M (1999) Immunomodulatory effects of tumor-associated fibroblasts in colorectaltumor development. Int J Cancer 81: 629-636.

Mcmillan DC (2013) The systemic inflammation-based Glasgow Prognostic Score: a decade of experience in patients with cancer. Cancer Treat Rev 39: 534-540.

Mocellin S, Marincola FM, Young HA (2005) Interleukin-10 and the immune response against cancer: a counterpoint. J Leukoc Biol 78: $1043-1051$.

Mohammed ZM, Going JJ, Edwards J, Elsberger B, Doughty JC, Mcmillan DC (2012) The relationship between components of tumour inflammatory cell infiltrate and clinicopathological factors and survival in patients with primary operable invasive ductal breast cancer. Br J Cancer 107: 864-873.

Movahedi K, Laoui D, Gysemans C, Baeten M, Stange G, Van Den Bossche J, Mack M, Pipeleers D, In't Veld P, De Baetselier P, Van Ginderachter JA (2010) Different tumor microenvironments contain functionally distinct subsets of macrophages derived from Ly6C(high) monocytes. Cancer Res 70: $5728-5739$.

Oliphant R, Nicholson G, Horgan P, Molloy R, Mcmillan D, Morrison D (2013) Deprivation and colorectal cancer surgery: longer-term survival inequalities are due to differential postoperative mortality between socioeconomic groups. Ann Surg Oncol 1-8.

Piancatelli D, Romano P, Sebastiani P, Adorno D, Casciani CU (1999) Local expression of cytokines in human colorectal carcinoma: evidence of specific interleukin-6 gene expression. J Immunother 22: 25-32.

Pollheimer MJ, Kornprat P, Lindtner RA, Harbaum L, Schlemmer A, Rehak P, Langner C (2010) Tumor necrosis is a new promising prognostic factor in colorectal cancer. Hum Pathol 41: 1749-1757.

Richards CH, Flegg KM, Roxburgh CS, Going JJ, Mohammed Z, Horgan PG, Mcmillan DC (2012a) The relationships between cellular components of the peritumoural inflammatory response, clinicopathological characteristics and survival in patients with primary operable colorectal cancer. Br J Cancer 106: 2010-2015.

Richards CH, Roxburgh CS, Anderson JH, Mckee RF, Foulis AK, Horgan PG, Mcmillan DC (2012b) Prognostic value of tumour necrosis and host inflammatory responses in colorectal cancer. Br J Surg 99: 287-294.

Richards CH, Roxburgh CS, Macmillan MT, Isswiasi S, Robertson EG, Guthrie GK, Horgan PG, Mcmillan DC (2012c) The relationships between body composition and the systemic inflammatory response in patients with primary operable colorectal cancer. PLoS One 7: e41883.

Richards DM, Hettinger J, Feuerer M (2012d) Monocytes and macrophages in cancer: development and functions. Cancer Microenviron. e-pub ahead of print 24 November 2012.

Rose-John S, Waetzig GH, Scheller J, Grotzinger J, Seegert D (2007) The IL-6/ sIL-6R complex as a novel target for therapeutic approaches. Expert Opin Ther Targets 11: 613-624.

Roxburgh CS, Mcmillan DC (2012) The role of the in situ local inflammatory response in predicting recurrence and survival in patients with primary operable colorectal cancer. Cancer Treat Rev 38: 451-466.

Scheller J, Ohnesorge N, Rose-John S (2006) Interleukin-6 trans-signalling in chronic inflammation and cancer. Scand J Immunol 63: 321-329. 
Sica A, Schioppa T, Mantovani A, Allavena P (2006) Tumour-associated macrophages are a distinct M2 polarised population promoting tumour progression: potential targets of anti-cancer therapy. Eur J Cancer 42: 717-727.

Solinas G, Germano G, Mantovani A, Allavena P (2009) Tumor-associated macrophages (TAM) as major players of the cancer-related inflammation. J Leukoc Biol 86: 1065-1073.

Strassmann G, Fong M, Kenney JS, Jacob CO (1992) Evidence for the involvement of interleukin 6 in experimental cancer cachexia. J Clin Invest 89: 1681-1684.

Trikha M, Corringham R, Klein B, Rossi JF (2003) Targeted anti-interleukin-6 monoclonal antibody therapy for cancer: a review of the rationale and clinical evidence. Clin Cancer Res 9: 4653-4665.
Zhou Q, Peng RQ, Wu XJ, Xia Q, Hou JH, Ding Y, Zhou QM, Zhang X, Pang ZZ, Wan DS, Zeng YX, Zhang XS (2010) The density of macrophages in the invasive front is inversely correlated to liver metastasis in colon cancer. J Transl Med 8: 13.

This work is published under the standard license to publish agreement. After 12 months the work will become freely available and the license terms will switch to a Creative Commons AttributionNonCommercial-Share Alike 3.0 Unported License. 